\title{
POSSIBILITIES AND LIMITATIONS OF OFF-RESONANCE POLARIZATION SENSITIVE CARS OF SHORT CHAIN PROTEINS
}

\author{
T.A.H.M. SCHOLTEN, G.W. LUCASSEN, F.F.M. DE MUL and J. GREVE \\ University of Twente, Dept. Applied Physics, PO-box 217, 7500 AE Enschede, The Netherlands
}

Received 16 January 1989

\begin{abstract}
Polarization sensitive CARS in the absence of resonance enhancement is applied to a short chain protein. The minimum concentration to record polarization sensitive CARS spectra of protein solutions is estimated to be $10 \mathrm{mg} / \mathrm{ml}$. The effects limiting the protein concentration are discussed and regarded from an experimental point of view. Signal strength and line parameters of polarization sensitive CARS spectra of the short chain protein Lysyl-Tryptophyl-Lysine are compared with those of a normal Raman spectrum.
\end{abstract}

\section{Introduction}

Generally resonance enhancement is used in CARS measurements (coherent anti-Stokes Raman scattering) of biophysically interesting molecules to obtain sufficient discrimination against the nonresonant background generated by the solvent [1]. We here discuss the possibilities and limitations of polarization sensitive CARS (PCARS) [2] for the suppression of nonresonant background of a solution of a model compound for proteins, in the absence of resonance enhancement.

A short chain structure of three amino acids is used as a protein model. It consists of two L-Lysine and one L-Tryptophan molecule. The choice of LysylTryptophyl-Lysine (Lys-Trp-Lys) as a sample molecule for non-electron resonant PCARS measurements were determined mainly by the availability of a normal Raman spectrum, enabling direct comparison.

\section{Experimental}

Spectra were recorded using the CARS spectrometer previously described in literature [3]. The 532 $\mathrm{nm}$ output of the doubled YAG laser ( $8 \mathrm{~ns}$ pulse width and $10 \mathrm{~Hz}$ repetition rate) was used as the pump beam. The dye laser provided the Stokes beam, which was tunable from $800-1650 \mathrm{~cm}^{-1}$ using rho- damine $6 \mathrm{G}$ dye. The YAG laser was operated at 40 $\mathrm{J} /$ pulse flashlamp power. The energy incident on the sample was calculated to be $\approx 200 \mu \mathrm{J} /$ pulse for the pump and $\approx 150 \mu \mathrm{J} /$ pulse for the Stokes beam.

A detail of the set-up for polarization sensitive measurements is shown in fig. 1. The polarization orientation of the Stokes beam is perpendicular to the optical table and adjusted for minimum depolarization caused by the matching mirror. The polarization orientation of the pump beam is $\phi=60.0^{\circ}$ with respect to the polarization direction of the Stokes beam. For this angle the vibration resonant PCARS signal is maximal [4]. The Glan-Taylor polarization analyzer, which is positioned directly behind the sample, is rotated to obtain a minimum nonresonant background. The perpendicular-to-parallel transmission ratio of the pump beam polarizer and signal analyzer is $9.5 \times 10^{-8}$ at $532 \mathrm{~nm}$. For the Stokes beam polarizer and signal analyzer this figure is $1.2 \times 10^{-7}$ at $560 \mathrm{~nm}$. These figures were measured with the matching mirror and lens in place but in absence of the sample.

Lys-Trp-Lys ( $25 \mathrm{mg}$ acetate salt, manufactured by Sigma) was dissolved in $0.5 \mathrm{ml}$ destilled water. The sample was contained in a $2 \mathrm{~mm}$ pathlength cell with $0.13 \mathrm{~mm}$ thick cover glass windows. The cover glasses were antireflection coated on their air/glass boundary [3]. The molecular weight of Lys-Trp-Lys is 593 


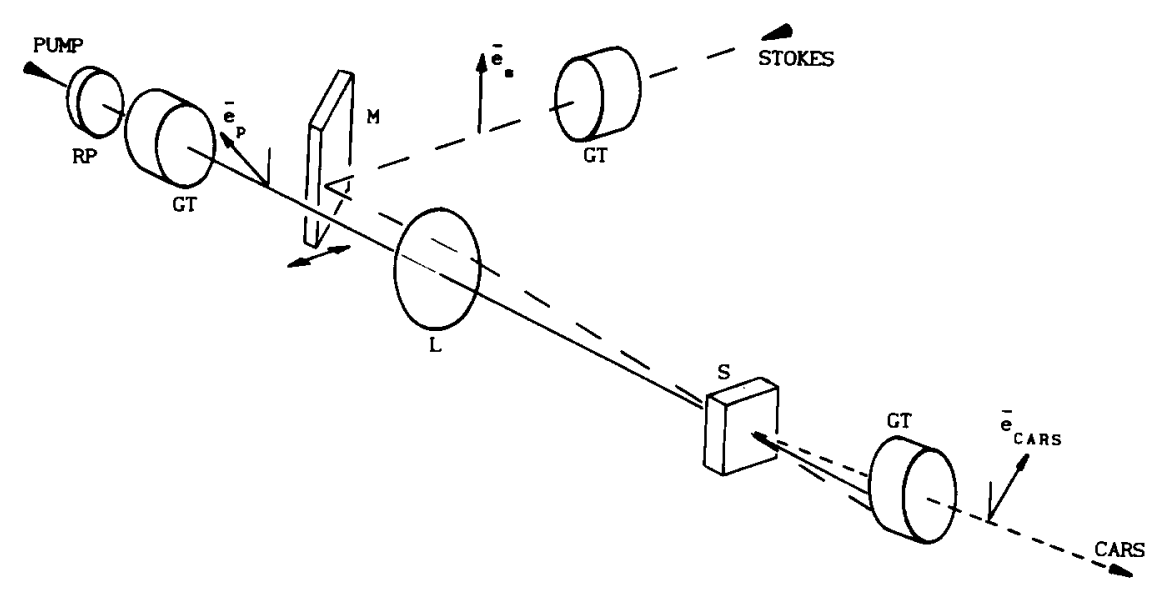

Fig. 1. Detail of the set-up polarization sensitive measurements. GT: Glan-Taylor polarizer; RP: half-wave retardation plate; M: matching mirror; L: lens ( $250 \mathrm{~mm}$ ); S: sample. The matching mirror can be translated as indicated to obtain the correct phase-match angle between the pump and Stokes beams.

and includes two acetate groups $\left(\mathrm{CH}_{3} \mathrm{COO}\right)$ which were added as counter ions.

Each presented PCARS spectrum consists of two added scans of 300 spectral points with 25 pulses averaged per point. Furthermore, the spectra are smoothed by a 5-points smoothing procedure.

\section{Results and discussion}

The PCARS spectra of $50 \mathrm{mg} / \mathrm{ml}(=84 \mathrm{mMolar})$

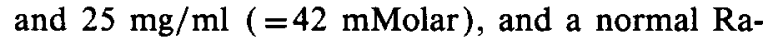
man spectra of $50 \mathrm{mg} / \mathrm{ml}$ Lys-Trp-Lys are shown in fig. 2. The analyzer orientation for minimal nonresonant background (at $1130 \mathrm{~cm}^{-1}$ ) is $\psi_{0}$ $=62.4^{\circ} \pm 0.3^{\circ}$, and deviates slightly from the $60^{\circ}$ predicted by Kleinman symmetry [5]. This indicates a nonresonant background depolarization ratio of $\rho^{\mathrm{NR}}=1 / 3.3$ instead of $1 / 3$. Deviations from Kleinman symmetry may occur in transparent media if a two-phonon absorption process of pump photons is not far from electronic resonance [6]. The achieved background reduction in the CARS spectrum, with respect to a spectral band with a depolarization $\rho^{\mathrm{R}}=0$, was measured to be $\approx 2 \times 10^{4}$. The sharp peak in the PCARS spectra at $1088 \mathrm{~cm}^{-1}$ is a calcite contribution caused by a minor overlap of the pump and Stokes beams, or reflections of these, in- side the calcite Glan-Taylor polarization analyzer. This CARS contribution showed to be additive, and not interfering with the sample spectrum.

A comparison of the positions of the vibrational bands in the PCARS spectra with those in the Raman spectrum show great similarity for most of the strongest modes. These bands mainly originate from the aromatic Tryptophan group. Clearly seen are the $882,931,1017 \mathrm{~cm}^{-1}$ lines, the 1242,1260 and 1346 , $1365 \mathrm{~cm}^{-1}$ doublets and the bands at 1447, 1466 , 1559,1584 and $1624 \mathrm{~cm}^{-1}$. More features can be found in the PCARS spectrum when heterodyning the vibration resonant signal with a controlled amount of nonresonant background. This situation and more on the spectral content of the PCARS spectra is discussed subsequently.

Note that Raman spectrum, contrary to the PCARS spectra, has a background, which is accounted for in the offset of the intensity axis of fig. $2 a$. This solvent background and its effect on the signal-to-noise ratio differs in several ways from the nonresonant background encountered in normal CARS spectra:

- The signal-to-background ratio is linear in sample concentration for Raman spectra and quadratic for CARS spectra (with respect to the lorentzian, i.e. vibration resonant contribution ).

- The Raman background does not interfere with the sample signal and can simply be subtracted. In 


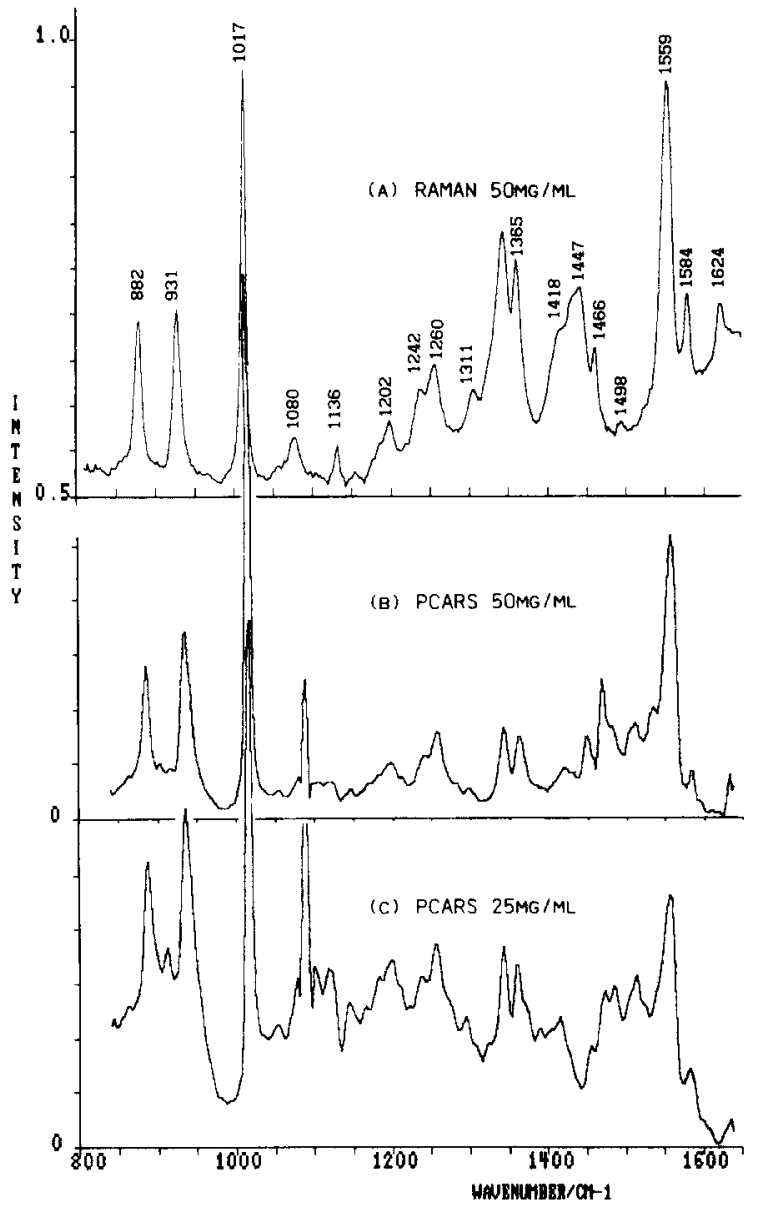

Fig. 2. (a) Normal Raman spectrum of $50 \mathrm{mg} / \mathrm{ml}$ Lys-Trp-Lys excited at $514 \mathrm{~nm}$. Intensity $=1.0$ corresponds with a Raman signal of $1.3 \times 10^{5}$ counts $/ \mathrm{s}$. (b) PCARS spectrum of $50 \mathrm{mg} / \mathrm{ml}$ LysTrp-Lys. Excitation at $532 \mathrm{~nm}$. (c) PCARS spectrum of $25 \mathrm{mg}$ / ml Lys-Trp-Lys. Excitation at $532 \mathrm{~nm}$.

case of CW excitation the signal-to-noise ratio of the subtracted spectrum is generally limited by photon shot noise of the background. The CARS nonresonant background interferes with the vibration resonant signal giving dispersive spectral bands. The intensities of these dispersive bands are linear in sample concentration. However, background subtraction is (despite from obtaining a dispersive spectrum) ultimately limited by laser-induced pulse-to-pulse fluctuation in the background [3] rather than by photon shot noise.
- In theory the CARS nonresonant background can be fully suppressed using the polarization sensitive technique. This cannot be realized with any polarization configuration for the background in the Raman spectrum.

The intensity of the $1017 \mathrm{~cm}^{-1}$ Tryptophan vibration in the Raman spectrum is $\sim 5 \times 10^{4}$ counts/ s. Thus, assuming a photocathode efficiency of 0.1 and a monochromator throughput of $0.25, \sim 2 \times 10^{6}$ photons/s are collected from the sample. Since the Raman spectrum was recorded in a macroscopical set-up with a low signal collection angle aperture, the total number of isotropically scattered photons was a factor $\sim 10$ higher. For the $50 \mathrm{mg} / \mathrm{ml}$ PCARS spectrum the number of scattered photons is $\approx 2 \times 10^{5}$ photons/pulse or $2 \times 10^{6}$ photons/s. Disregarding the difference in irradiating energy (a factor 10-100 higher in case of Raman scattering) which is inherent with these techniques, the PCARS signal is comparable in magnitude with the Raman signal. This relatively low CARS signal results from the quadratic decrease of the signal with the sample concentration.

Upon reducing the concentration from 50 to 25 $\mathrm{mg} / \mathrm{ml}$ (a factor 4 in vibration resonant contribution) the leakage of nonresonant background becomes noticeable (fig. 2c). A further decrease in sample concentration is limited since the signal-tononresonant background ratio reduces quadratically. Some improvement in background suppression can be gained by repeatedly adjusting the Stokes beam polarization orientation and analyzer setting for minimum background signal. Residual birefringence in cell windows is then compensated by introducing an opposite ellipticity of the Stokes beam which results upon reflection by the matching mirror. Also, an improvement of 33\% in signal-to-background ratio can be obtained at the cost of $20 \%$ of the vibration resonant signal, by selecting $\phi=71.6^{\circ}$ instead of $\phi=60^{\circ}$ [4].

Furthermore, the vibration resonant signal reduces quadratically with concentration, which eventually hampers the detection. This can be compensated by increasing the pump intensity and/or increasing the sample thickness. In the first situation the signal-to-background ratio remains the same; the pump intensity is ultimately limited by sample heating, dielectric breakdown and/or photo-degrada- 
tion. When increasing the sample thickness, however, the signal-to-background ratio decreases: The additional length of the sample is excited by a pump and Stokes beam which are already partly depolarized by the first (original) sample layer. It therefore generates a CARS signal with a lower polarization quality, thus affecting the background suppression.

At low concentrations the analyzer angle setting becomes very critical, and slight deviations result in large effects on the spectral band shape. Furthermore, small variations in the value of the depolarization ratio of the solvent signal throughout the spectrum result in a quick increase in the background for wavenumber shifts outside the range where a minimum was obtained. In fig. 3 , two representative spectra of $10 \mathrm{mg} / \mathrm{ml}$ Lys-Trp-Lys are shown (same conditions as fig. 2 except that the

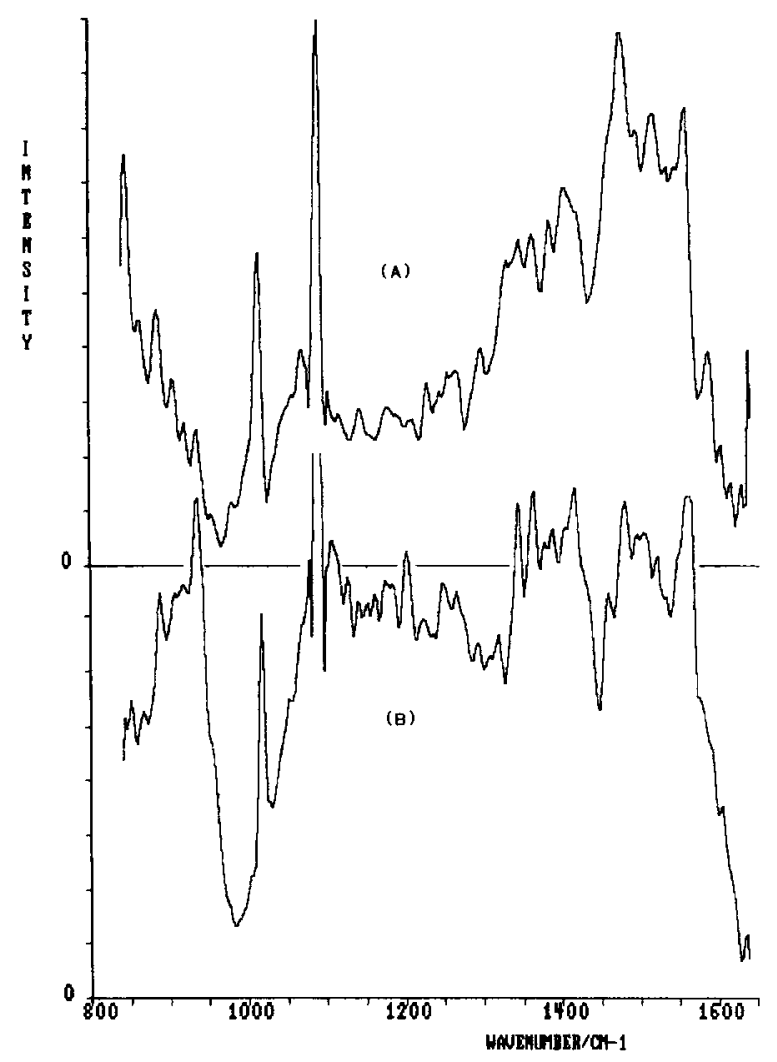

Fig. 3. PCARS spectra of $10 \mathrm{mg} / \mathrm{ml}$ Lys-Trp-Lys. Excitation at $532 \mathrm{~nm}$; background minimized at $1130 \mathrm{~cm}^{-1}$ (a) and $970 \mathrm{~cm}^{-1}$ (b). flashlamp power is increased to $45 \mathrm{~J} /$ pulse). According to the PCARS spectra of fig. 2, one spectrum was minimized at $970 \mathrm{~cm}^{-1}$, the other at $1130 \mathrm{~cm}^{-1}$. Large differences result from the varying suppression of the background, and thus varying dispersive contributions throughout the spectrum. Although multiple scans reproduced very well, it showed to be difficult to obtain the same spectral shape when starting off again. However, when comparing with fig. 2, the main spectral features of Lys-Trp-Lys can still be distinguished in the $10 \mathrm{mg} / \mathrm{ml}$ spectra.

According to the previous discussion the estimated concentration limit for recording nonresonantly excited PCARS spectra of proteins is estimated to be approximately $10 \mathrm{mg} / \mathrm{ml}$. It should be noted that this figure is obtained from measurement of a model compound containing 1/3 Tryptophan and $2 / 3$ Lysine. For molecules with a lower concentration of a certain group the $10 \mathrm{mg} / \mathrm{ml}$ must accordingly be increased to be able to record the spectral contributions from that specific group.

The spectral information in a PCARS spectrum is affected by the depolarization ratios of the vibrations: Vibrations with depolarization ratios close to $\rho^{\mathrm{NR}}$ are not or hardly visible in the PCARS spectrum. Besides this $\rho^{\mathrm{R}}$ dependence, the intensity of the lorentzian spectral bands in the PCARS spectra depends quadratically on the Raman scattering crosssection. This is in contrast with the dispersive interference with the nonresonant background in normal CARS, which results in a linear relationship [7]. Spectral contributions with a small scattering crosssection and/or a depolarization ratio close to $\rho^{\mathrm{NR}}$, are therefore more easily detected when a controlled amount of nonresonant signal is mixed with the vibration resonant signal.

This heterodyning effect is shown in fig. 4 for the $50 \mathrm{mg} / \mathrm{ml}$ Lys-Trp-Lys sample: The analyzer angle is offset by $\Delta \psi= \pm 0.55^{\circ}$ from the angle $\psi_{0}$. The spectra have the same vertical scale. The nonresonant background (approximately given by the mean level in the $1100-1400 \mathrm{~cm}^{-1}$ range) increases symmetrically around $\psi_{0}$, from nearly zero at $\psi_{0}$ to $\sim 0.5$ at $|\Delta \psi|=0.55^{\circ}$ and $\sim 1.6$ at $|\Delta \psi|=1.1^{\circ}$ (not shown), relative to the vibration resonant intensity of the 1017 $\mathrm{cm}^{-1}$ band. Weak vibrations become visible as dispersive bands with opposite sign on either side of $\psi_{0}$. The sign of a dispersive contribution and the sign of 


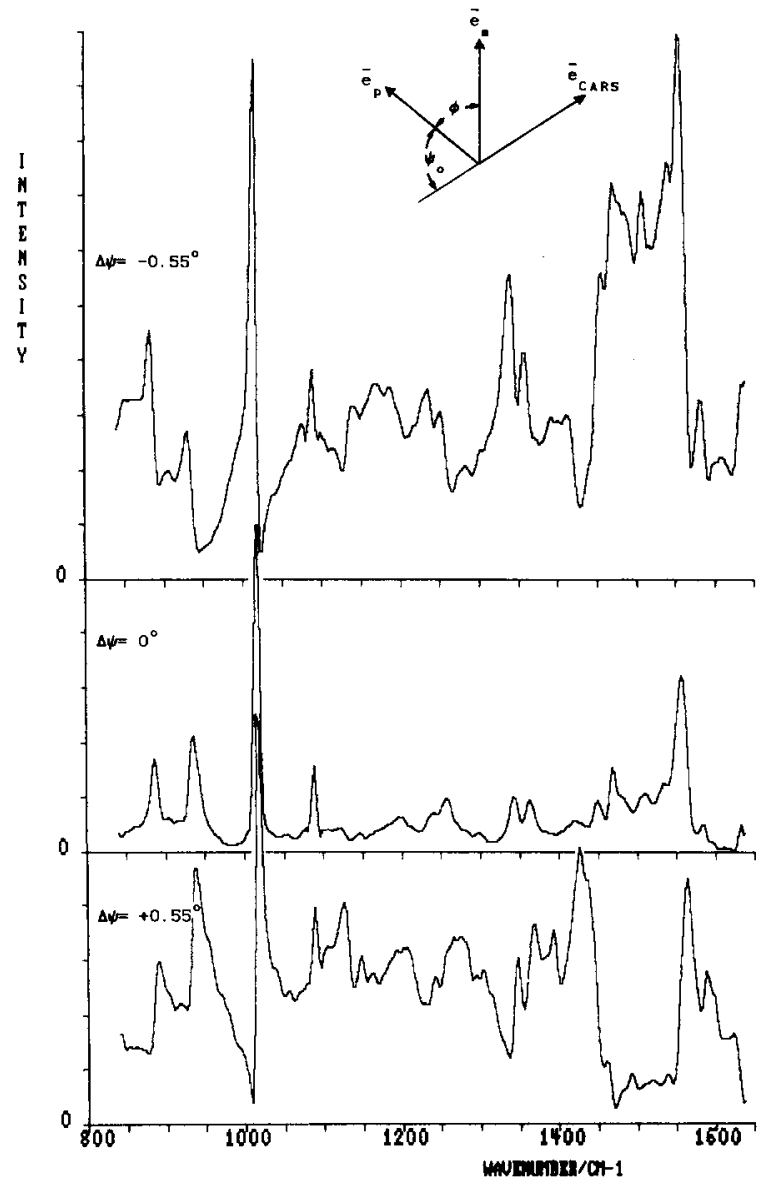

Fig. 4. PCARS spectra of $50 \mathrm{mg} / \mathrm{ml}$ Lys-Trp-Lys. From top to bottom the angle $\psi_{0}$ for minimal background is varied by $-0.55^{\circ}$, $0^{\circ}$ and $+0.55^{\circ}$. The spectra have the same vertical scale. Inset: polarization orientation of the pump $\left(\bar{e}_{\mathrm{p}}\right)$, Stokes $\left(\bar{e}_{\mathrm{s}}\right)$ and CARS $\left(\bar{e}_{\mathrm{CARS}}\right)$ beams.

$\Delta \psi$ determine whether the depolarization $\rho^{\mathrm{R}}$ of the vibration is smaller or larger than $\rho^{\mathrm{NR}}$. This qualitative relationship is given in table 1 . If the difference in the intensity of the dispersive contribution of the vibrations at angle offsets $+\Delta \psi$ and $-\Delta \psi$ is large, then $\rho^{\mathrm{R}}$ is close to $\rho^{\mathrm{NR}}$.

The depolarization ratios of all bands in the LysTrp-Lys spectra deviate significantly from the depolarization ratio of the nonresonant background. Fig. 5 shows simulated spectra using equations for the PCARS signal derived by Brakel et al. [4] and assuming that for all bands the depolarization is either $\rho^{\mathrm{R}}=0$ (i.e. polarized, for bands which are positive dispersive for $\Delta \psi<0$ ) or $\rho^{\mathrm{R}}=0.75$ (i.e. depo-
Table 1

Qualitative determination of the depolarization ratio $\rho^{\mathrm{R}}$ from the sign of the dispersive contribution in a PCARS spectrum recorded with an analyzer angle offset $\Delta \psi$. The lineshape $\delta /$ $\left(\delta^{2}+\Gamma^{2}\right)$, with $\delta=\omega_{\text {vibr }}-\left(\omega_{\mathrm{p}}-\omega_{\mathrm{s}}\right)$ the detuning of the pump $\left(\omega_{\mathrm{p}}\right)$ and Stokes $\left(\omega_{\mathrm{s}}\right)$ frequencies from the vibrational frequency $\omega_{\text {vibr }}$ and $\Gamma$ the HWHM of the vibration, is defined as positive dispersive.

\begin{tabular}{|c|c|c|c|}
\hline & \multicolumn{3}{|l|}{ Dispersion } \\
\hline & + & 0 & - \\
\hline$\Delta \psi>0$ & $\rho^{\mathrm{R}}>\rho^{\mathrm{NR}} A$ & $\rho^{\mathrm{R}}=\rho^{\mathrm{NR}} A$ & $\rho^{\mathrm{R}}<\rho^{\mathrm{NR}} A$ \\
\hline$\Delta \psi<0$ & $\rho^{\mathrm{R}}<\rho^{\mathrm{NR}} A$ & $\rho^{\mathrm{R}}=\rho^{\mathrm{NR}} A$ & $\rho^{\mathrm{R}}>\rho^{\mathrm{NR}} A$ \\
\hline \multicolumn{4}{|c|}{$A=\frac{\tan \left(\psi_{0}\right)}{\tan \left(\psi_{0}+\Delta \psi\right)}$} \\
\hline
\end{tabular}

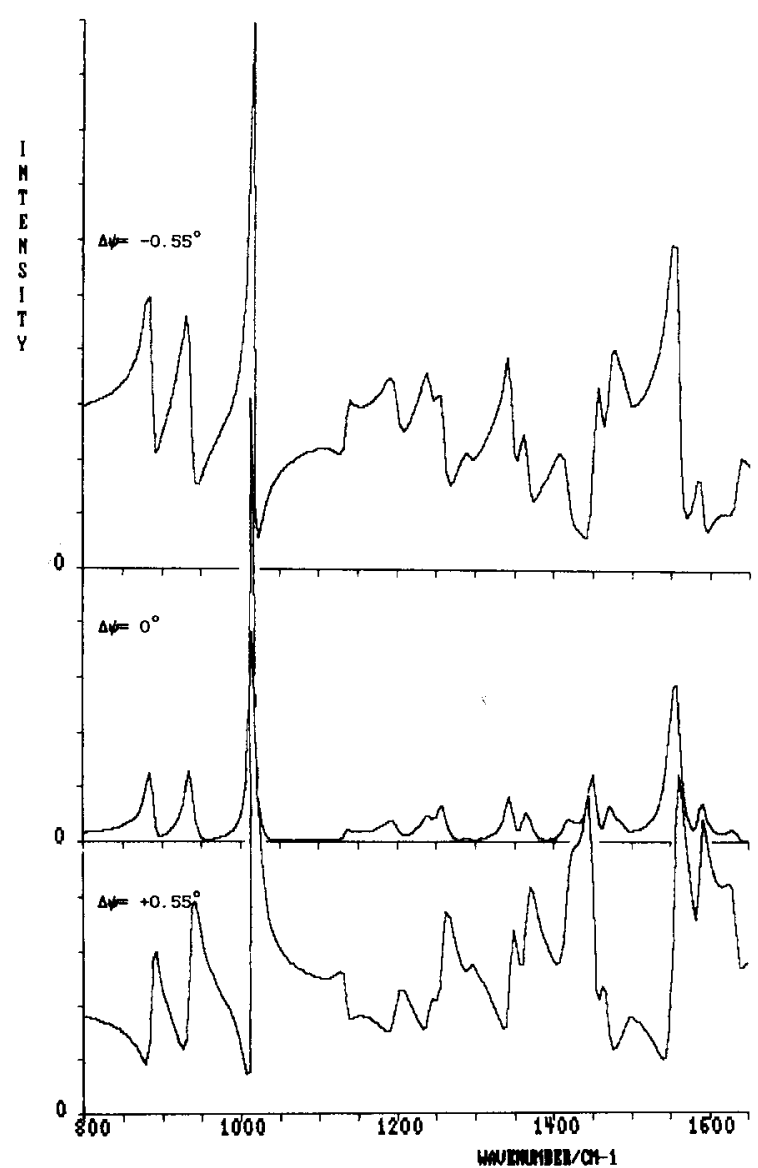

Fig. 5. Simulated PCARS spectra using the line parameters summarized in table 2 . From top to bottom the angle $\psi_{0}$ is varied by $-0.55^{\circ}, 0^{\circ}$ and $+0.55^{\circ}$. For the calculation a frequency independent nonresonant background susceptibility $\chi_{1111}^{\mathrm{NR}}$ of 25 times the $\chi_{1111}^{\mathrm{R}}$ magnitude of the $1014 \mathrm{~cm}^{-1}$ band was used. 
Table 2

Vibrational wavenumbers, $\chi_{1111}^{\mathrm{R}}$ peak magnitudes, depolarization ratios and approximate linewidths of Lys-Trp-Lys in the 800-1650 $\mathrm{cm}^{-1}$ range as determined from the PCARS spectra and simulations of figs. 4 and 5 . A depolarization ratio of 0 or 0.75 is assumed for the vibrational bands. This indicates that vibrational depolarization ratios deviate significantly from the background depolarization ratio $(\sim 0.3)$ (see text).

\begin{tabular}{|c|c|c|c|c|}
\hline $\begin{array}{l}\text { Band position } \\
\text { Raman spectrum }\end{array}$ & $\begin{array}{l}\text { Band position } \\
\text { PCARS spectra }\end{array}$ & $\begin{array}{l}\chi_{1111}^{\mathrm{R}} \\
\text { (relative) }\end{array}$ & $\begin{array}{l}\text { magn. } \\
\text { depolarization } \\
\text { ratio }\end{array}$ & $\begin{array}{l}\text { HWHM } \\
\Gamma\end{array}$ \\
\hline $882 \mathrm{~m}$ & $884 m$ & 3.3 & 0 & 5.5 \\
\hline $931 \mathrm{~m}$ & $934 m$ & 3.7 & 0 & 7 \\
\hline $1017 \mathrm{~s}$ & $1014 \mathrm{~s}$ & 10 & 0 & 3 \\
\hline $1080 w$ & (overlap 1088?) & & & \\
\hline $1136 \mathrm{w}$ & $1132 \mathrm{vw}$ & 0.7 & 0.75 & 5.5 \\
\hline $1154 \mathrm{vw}$ & - & & & \\
\hline $1202 w$ & $1197 w$ & 1.3 & 0 & 10 \\
\hline $1242 \mathrm{w}, \mathrm{sh}$ & $1240 \mathrm{w}$ & 1.3 & 0 & 8 \\
\hline \multirow[t]{2}{*}{$1260 \mathrm{~m}$} & $1257 \mathrm{~m}$ & 2.5 & 0 & 8 \\
\hline & $1291 \mathrm{vw}$ & 0.5 & 0 & 8 \\
\hline \multicolumn{5}{|l|}{$1311 w$} \\
\hline $1346 \mathrm{~m}$ & $1343 \mathrm{~m}$ & 2.7 & 0 & 6 \\
\hline $1365 \mathrm{~m}$ & $1364 \mathrm{~m}$ & 2.2 & 0 & 7.5 \\
\hline $1418 \mathrm{w}, \mathrm{sh}$ & $1415 \mathrm{~m}$ & 2.0 & 0 & 10 \\
\hline $1447 \mathrm{~m}$ & $1449 m$ & 2.7 & 0.75 & 6 \\
\hline $1466 \mathrm{mw}$ & $1468 w$ & 1.3 & 0.75 & 6 \\
\hline $1498 \mathrm{vw}$ & $1491 \mathrm{vw}$ & 0.5 & 0 & 8 \\
\hline $1559 \mathrm{~s}$ & $1555 \mathrm{vs}$ & 6 & 0 & 9 \\
\hline $1584 \mathrm{mw}$ & $1587 \mathrm{~m}$ & 2.1 & 0 & 5 \\
\hline $1624 w$ & $1632 w$ & 1.0 & 0.75 & 6 \\
\hline
\end{tabular}

$\mathrm{v}=$ very, $\mathrm{s}=$ strong, $\mathrm{m}=$ medium, $\mathrm{w}=$ weak, $\mathrm{sh}=$ shoulder.

larized, for bands which are negative dispersive for $\Delta \psi<0$ ). The shapes of the simulated spectra agree with the measured spectra. The spectra were fitted by hand; a better fit can be obtained by a recursive computer procedure which, however, was not available to us. Due to the opposed sign of $\left(\rho^{\mathrm{R}}-\rho^{\mathrm{NR}}\right)$ for the 1415 and the $1449,1468 \mathrm{~cm}^{-1}$ vibrations, these bands are easily resolved (comparison to the Raman spectrum). It demonstrates the possibility to yield more structure in overlapping spectral bands by using the polarization sensitive CARS technique [8].

Table 2 summarizes the vibrational wavenumbers found in the PCARS spectra and the Raman spectrum, and gives $\chi_{1111}^{\mathrm{R}}$ magnitudes and $\rho^{\mathrm{R}}$ values which were used for the simulated spectra of fig. 5. It should be noted that the determination of $\rho^{\mathrm{R}}$ values, and coupled to this the $\chi_{111}^{\mathrm{R}}$ magnitude from a set of spectra with $\Delta \psi$ small, is only accurate for $\rho^{\mathrm{R}}$ close to $\rho^{\mathrm{NR}}$. Then, the line contour and intensity change significantly with $\Delta \psi$. The relative change $\Delta I^{\mathrm{R}} / I^{\mathrm{R}}$ of a vibration resonant contribution to the CARS spec- trum, upon changing the analyzer angle offset from $\Delta \psi_{0}$ to $-\Delta \psi_{0}$, can be written as

$\frac{\Delta I^{\mathrm{R}}}{I^{\mathrm{R}}}=\frac{4 \tan \left(\Delta \psi_{0}\right) \tan \left(\left|\theta^{\mathrm{R}}-\theta^{\mathrm{NR}}\right|\right)}{\tan ^{2}\left(2 \Delta \psi_{0}\right)+\tan ^{2}\left(\theta^{\mathrm{R}}-\theta^{\mathrm{NR}}\right)}$,

where $\theta^{\mathrm{R}}$ and $\theta^{\mathrm{NR}}$ are the angles between the polarization orientation $\bar{e}_{\mathrm{p}}$ of the pump beam and the polarization orientation of the vibration resonant and nonresonant signals, respectively. These are given by

$\theta^{\mathrm{R}}=\arctan \left[\rho^{\mathrm{R}} \tan (\phi)\right]$,

$\theta^{\mathrm{NR}}=\arctan \left[\rho^{\mathrm{NR}} \tan (\phi)\right]$.

With $\rho^{\mathrm{NR}}=1 / 3.3, \phi=60^{\circ}$ and $\Delta \psi_{0}=0.55^{\circ}$ this gives a relative change of $\sim 8 \%$ for vibrations with $\rho^{R}=0$ or 0.75 and $\sim 30 \%$ for $\rho^{\mathrm{R}}=0.22$ or 0.40 . If the accuracy with which the vibration resonant contribution in the spectra with $\Delta \psi_{0}= \pm 0.55^{\circ}$ can be estimated is within $30 \%$ (mainly determined by pulseto-pulse fluctuations on the nonresonant back- 
ground and fluctuations during scanning), only depolarization ratios in the $\rho^{\mathrm{R}}=0.2-0.4$ range can be determined accurately. Therefore, the $\rho^{\mathrm{R}}$ values of 0 and 0.75 should be read as $\rho^{\mathrm{R}} \ll \rho^{\mathrm{NR}}$ and $\rho^{\mathrm{R}} \gg \rho^{\mathrm{NR}}$, respectively. A change of $\rho^{\mathrm{R}}$ from $0 \rightarrow 0.2$ or $0.75 \rightarrow 0.5$ combined with a factor 2.5 increase of the $\chi_{1111}^{\mathrm{R}} \mathrm{mag}$ nitude, and thus keeping $\chi_{1111}^{\mathrm{R}} \times\left(1-3 \rho^{\mathrm{R}}\right)$ constant [4], only results in minor changes in the simulated spectra.

With exception of the very weak $1154 \mathrm{~cm}^{-1}$ band and the $1080 \mathrm{~cm}^{-1}$ band (which is overlapping with the $1088 \mathrm{~cm}^{-1}$ band of the calcite polarizer), all bands observable in the Raman spectrum are present in the PCARS spectra. Discrepancy is present in the position of the $1311 \mathrm{~cm}^{-1}$ (Raman) versus 1291 $\mathrm{cm}^{-1}$ (PCARS) band. Although strongly depending on the exact value of the depolarization ratio $\rho^{\mathrm{R}}$, the $\chi_{1111}^{\mathrm{R}}$ magnitude, which is proportional to the Raman scattering cross-section, is on the basis of the quantitative indication ( $\mathrm{s}, \mathrm{m}, \mathrm{w}$, etc.) comparable with the Raman band intensity.

\section{Conclusion}

In the general case of PCARS applied to nonresonantly excited (transparent) solutions of biological proteins and other molecules, it can be concluded from the above presented results, that:

- The minimum sample concentration lies in the $10-20 \mathrm{mg} / \mathrm{ml}$ range (20-50 mMolar) and is limited by the maximum achievable suppression of the nonresonant background and the intensity of the CARS signal, which decreases quadratically with sample concentration. Due to this quadratic dependence, the PCARS signal strength for the minimum sample concentration is of the same order as the normal Raman signal. To improve the detection limit towards and below the mMolar range, resonance enhancement is required.

- Using the PCARS technique, overlapping bands may be resolved when the depolarization ratios of the overlapping vibrations are different. A qualitative indication for the vibrational depolarization ratios with respect to the depolarization ratio of the nonresonant background can be obtained from a spectrum recorded with $\Delta \psi \neq 0$ and is determined by the sign of the dispersive line contours (table 1 ). The accuracy of the determination of $\rho^{\mathrm{R}}$ and $\chi_{1111}^{\mathrm{R}}$ from a set of PCARS spectra with $\Delta \psi$ small is best for $\rho^{\mathrm{R}}$ close to $\rho^{\mathrm{NR}}$.

- The PCARS technique essentially gives the same information as can be obtained with the normal $\mathrm{Ra}$ man technique. Therefore, apart from resolving overlapping vibrations, the PCARS technique is favoured for transparent media in case fluorescence interferes with the Raman measurement.

In case of large chain molecules, depolarization by the macromolecules obstructs the suppression of the nonresonant background. As a result of this, no PCARS spectra could be recorded of a $50 \mathrm{mg} / \mathrm{ml}$ DNA solution. When lowering the concentration the depolarization reduces and the background suppression improves. The improvement, however, is offset by the quadratic decrease of the vibration resonant signal in comparison with the solvent background. When reducing the sample thickness, the depolarization may also be lowered while keeping the concentration constant. In this way, although at the cost of CARS signal intensity, we were able to record CARS spectra of $\sim 100 \mu \mathrm{m}$ slides of fluoresceing human eye lenses which contain an approximately $1: 3$ protein/water weight-ratio. We hope to report on these measurements soon.

\section{References}

[1] F.W. Schneider, in: Non-linear Raman spectroscopy and its chemical applications, eds. W. Kiefer and D.A. Long (D. Reidel, Holland 1982) p. 445.

[2] S.A. Akhmanov, A.F. Bunkin, S.G. Ivanov and N.I. Koroteev, Sov. Phys. JETP 47 (1978) 667.

[3] T.A.H.M. Scholten, G.W. Lucassen, F.F.M. de Mul and J. Greve, Appl. Optics 27 (1988) 3225.

[4] R. Brakel, V. Mudogo and F.W. Schneider, J. Chem. Phys. 84 (1986) 2451.

[5] D.A. Kleinman, Phys. Rev. 126 (1962) 1977.

[6] M.D. Levenson and N. Bloembergen, Phys. Rev. B 10 (1974) 4447.

[7] W.M. Tolles, J.W. Nibler, J.R. McDonald and A.B. Harvey, Appl. Spectrosc. 31 (1977) 253.

[8] A.F. Bunkin, D.V. Vlasov, A.S. Galumyan, D.V. Mal'tsev, and K.O. Surskii, Sov. J. Quantum Electron. 15 (1985) 513. 